\title{
A New Stationary Phase Prepared from Ground Silica Monolith Particles by Reversible Addition-Fragmentation Chain Transfer Polymerization
}

\author{
Seung Mi Lee, Shabi Abbas Zaidi, and Won Jo Cheong* \\ Department of Chemistry, Nano Fine Center, and Institute of Basic Research, Inha University, Incheon 402-751, Korea \\ "E-mail: wjcheong@inha.ac.kr \\ Received July 19, 2010, Accepted August 31, 2010
}

\begin{abstract}
Silica monolith powders were prepared by a new procedure where ground powders of proper size distribution were obtained without sieving. An initiator was attached to this ground monolith and polystyrene was bound by reversible addition-fragmentation chain transfer polymerization to give a new stationary phase. The separation efficiency of this phase was found better than that of the polystyrene bound phase based on conventional silica particles and that of the $\mathrm{C} 18$ bound silica monolith powders.
\end{abstract}

Key Words: Ground silica monolith particles, RAFT polymerization, Polystyrene bound phase, High separation efficiency

\section{Introduction}

Surface-attached initiators have been recently used to form controlled polymer layers on porous or nonporous inorganic surface and the resultant organic-inorganic combined polymer can be used as stationary phases in chromatography. ${ }^{1-16}$ Various silica materials including porous silica particles, ${ }^{1,4,6-7,11-13,14-16}$ silica beads, ${ }^{2,5,10}$ silica monolith, ${ }^{9}$ and silica capillary ${ }^{3,8}$ have been used for such purposes. Atom transfer radical polymerization (ATRP) was most frequently used in such studies ${ }^{1-10,16}$ while reversible addition-fragmentation chain transfer (RAFT) polymerization was also sometimes utilized. ${ }^{11-12,14-15}$ It is interesting to note that an initiator was attached to organic monolith particles instead of silica, and a polymer layer with ion exchange capability was added by ATRP in a specific study. ${ }^{17}$

The details of principles and applications of ATRP are referred to some review articles. ${ }^{18-19}$ Briefly, a catalytic mixture of cuprous/cupric halides and an amine base is used to activate polymerization on the silica surface with a halogen-containing initiator. In some of the ATRP studies, ${ }^{16}$ the resultant stationary phases were reported to show better separation selectivities than conventional $\mathrm{C} 18$ phases. However, the reported separation efficiencies of the stationary phases were yet considerably inferior to those of commercial C18 phases.

In RAFT polymerization, the initiator is made by attaching a halogen containing ligand to silica surface followed by reaction with sodium diethyldithiocarbamate as introduced in some review articles. ${ }^{20-21}$ According to the RAFT polymerization mechanism, ${ }^{20-21}$ the polymer chain is grown in the $\mathrm{C}-\mathrm{S}$ bond located between the spacer moiety and the diethyldithiocarbamate moiety of the initiator silica. The resultant stationary phases, however, showed inferior separation efficiencies ${ }^{11-12,1415}$ in comparison with the conventional $\mathrm{C} 18$ phases.

In our previous studies, we reported some initiator silica particles and new stationary phases prepared thereof. ${ }^{22-24}$ The initiator silica particles prepared by reacting silica particles with 3-chloropropyltrimethoxysilane followed by sodium diethyldithiocarbarmate resulted in stationary phases with improved morphology and better separation performance ${ }^{23-24}$ compared to the initiator silica particles prepared by reacting with 4-(chloromethyl)phenylisocyanate. ${ }^{22}$

In the above studies, commercial silica particles were used. We have been studying about ground silica monolith particles and their application ${ }^{25-26}$ in our laboratory. It has been demonstrated that the $\mathrm{C} 18$ modified ground monolith particles showed better separation efficiency than the $\mathrm{C} 18$ phase of conventional silica particles. ${ }^{25-26}$

In this study, ground silica monolith particles were prepared and reacted with 3-chloropropyltrimethoxysilane followed by sodium diethyldithiocarbarmate. The preparation method of ground silica monolith was modified and improved to leave out the tedious sieving process. The porous initiator monolith particles were then allowed to undergo radical polymerization with styrene to give a new stationary phase. This stationary phase was packed in a glass-lined stainless steel microcolumn to examine the chromatographic performance. We were able to attain more improved separation efficiency (number of theoretical plates) compared to the previous studies.

\section{Experimental}

Materials. Trimethylorthosilicate (TMOS), polyethylene glycol (PEG) 10000, glacial acetic acid, urea, styrene, 3-chloropropyltrimethoxysilane, sodium diethyldithiocarbamate, anhydrous tetrahydrofuran (THF), anhydrous toluene, anhydrous p-xylene, 1,1,1,3,3,3 hexamethyldisilazane (HMDS), trimethylchlorosilane (TMCS), chlorooctadecyldimethylsilane were purchased from Sigma-Aldrich (St. Louis, MO, USA). HPLC grade methanol, acetonitrile, water were purchased from Mallinckrodt Baker (Phillipsburg, NJ, USA). All the reagents were used as received. Screen frits (1.6 mm diameter, $0.08 \mathrm{~mm}$ thickness) were purchased from Valco (Houston, TX, USA). Pieces of glass lined stainless steel tubing ( $30 \mathrm{~cm}, 0.5 \mathrm{~mm}$ I.D, $1.6 \mathrm{~mm}$ O.D.) were purchased from Alltech (Deerfield, IL, USA).

Preparation of the ground monolith silica. The prior method $^{25-26}$ was modified to leave out the tedious sieving step. 
Thus $220 \mathrm{mg}$ PEG and $225 \mathrm{mg}$ urea were dissolved in $2.5 \mathrm{~mL}$ $0.01 \mathrm{~N}$ acetic acid in a vial. An ice/water bath was installed under the vial, and $1 \mathrm{~mL}$ TMOS was added and stirred for $40 \mathrm{~min}$. The vial was incubated at $40{ }^{\circ} \mathrm{C}$ for $48 \mathrm{hr}$, then at $120{ }^{\circ} \mathrm{C}$ for 48 $\mathrm{hr}$. Simultaneous reaction was taken for a group of vials $(\mathrm{n}>5)$. The formed monolith was taken out and ground smoothly by a mortar and pestle for $30 \mathrm{~min}$. The monolith powders were washed with 50/50 (v/v) 2-propanol/water at $120{ }^{\circ} \mathrm{C}$ for $24 \mathrm{hr}$ followed by $50 / 50 \mathrm{methanol} /$ water at $120^{\circ} \mathrm{C}$ for $24 \mathrm{hr}$, filtered, and dried at $60{ }^{\circ} \mathrm{C}$ for $24 \mathrm{hr}$. The monolith powders were finally calcined at $500{ }^{\circ} \mathrm{C}$ for $48 \mathrm{hr}$.

Synthesis of the initiator attached monolith silica. The initiator attached monolith silica was prepared by reacting the silica monolith particles with 3-chloropropyltrimethoxysilane followed by sodium diethyldithiocarbamate. First, the $500 \mathrm{mg}$ silica particles were dried at $120{ }^{\circ} \mathrm{C}$ for $4 \mathrm{~h}$ or more, and dispersed in a mixture of $2 \mathrm{~mL} 3$-chloropropyl trimethoxysilane and $8 \mathrm{~mL}$ anhydrous toluene. Reaction was carried out under reflux at $110{ }^{\circ} \mathrm{C}$ for $24 \mathrm{~h}$. After completion, the product was thoroughly washed with toluene followed by acetone, filtered, and air dried.

Next, $200 \mathrm{mg}$ sodium diethyldithiocarbamate was dissolved in $10 \mathrm{~mL}$ anhydrous $\mathrm{THF}$, and the solution was purged by nitrogen to remove oxygen, then the initiator-attached silica monolith particles were suspended in the solution and reacted at $55^{\circ} \mathrm{C}$ for $10 \mathrm{~h}$. The product was thoroughly washed with THF and acetone in sequence, filtered, dried and stored in vacuum desiccators at room temp.

The preparation scheme of the initiator attached silica monolith particles is shown in Scheme 1.

Polymerization of styrene upon the initiator attached silica. Styrene $2.5 \mathrm{~mL}$ was dissolved in $4 \mathrm{~mL}$ p-xylene, and the solution was purged by nitrogen, then the $100 \mathrm{mg}$ initiator silica particles were suspended by stirring, and radical polymerization was carried out under reflux at $110^{\circ} \mathrm{C}$ for $40 \mathrm{~h}$. The polystyrene combined silica particles were thoroughly washed with toluene, methanol, and acetone in sequence and dried at $60{ }^{\circ} \mathrm{C}$ for $10 \mathrm{~h}$.

C18 modification and end-capping of the ground monolith silica. An exact amount of $200 \mathrm{mg}$ ground monolith silica was modified with $0.12 \mathrm{~g}$ chlorodimethyloctadecylsilane dissolved in $5.0 \mathrm{~mL}$ anhydrous toluene in a small round bottom flask by stirring at $110^{\circ} \mathrm{C}$ for $10 \mathrm{~h}$, then encapped by adding the mixture of $105 \mu \mathrm{L}$ HMDS and $35 \mu \mathrm{L}$ TMCS slowly with a dropping funnel at $70{ }^{\circ} \mathrm{C}$ for $6 \mathrm{~h}$ or more. The final product was filtered and washed with toluene, methanol and acetone in sequence and dried at $60{ }^{\circ} \mathrm{C}$ for $10 \mathrm{~h}$.

Characterization of chromatographic phases. The BET/BJH nitrogen adsorption/ desorption isotherms were measured at 77 K using a BEL-Japan (Osaka, Japan) BELSORP-Max for the ground silica monolith, the initiator attached ground silica monolith, and the polystyrene attached ground silica monolith. The samples were out-gassed at $393 \mathrm{~K}$ for $10 \mathrm{~h}$ to obtain a residual pressure of less than $10^{-3}$ torr. The amount of $\mathrm{N}_{2}$ adsorbed at a relative pressure of $\mathrm{P} / \mathrm{P}_{0}=0.98$ was used to determine the total pore volume, which corresponded to the sum of the micro pore and meso pore volume. A HITACHI (Tokyo, Japan) S-4200 field emission scanning electron microscopy (FE-SEM) was used to obtain SEM images of the phases. A Thermo Electron (Waltham, MA, USA) Flash EA1112 elemental analyzer was used to obtain the carbon load data. A Malvern (Worcestershire, UK) Mastersizer 2000 particle size analyzer was used to measure the size distribution of the silica monolith particles.

Micro liquid chromatography $(\boldsymbol{\mu} \mathrm{LC})$. A Shimadzu (Tokyo, Japan) 10AD pump, a Shimadzu DGU-14A membrane degasser, a Valco (Houston, TX, USA) CI4W.05 injector with a $50 \mathrm{~nL}$ injection loop, a Jasco (Tokyo, Japan) UV-2075 UV-VIS capillary window detector, and the home-made $0.5 \mathrm{~mm}$ I.D. glasslined micro column were assembled to construct the $\mu \mathrm{LC}$ system. A PC system with the software Multichro 2000 from YoulinGisul (Sungnam, Korea) was used to acquire and process the chromatographic data.

The micro columns were packed according to the procedure reported before. ${ }^{22-24}$ Thus, a frit (commercial screen frit) was placed in the $1 / 16$ inch outlet of a $1 / 16-1 / 32$ reducing union, a piece of $30 \mathrm{~cm}$ glass lined stainless steel tubing was fitted to the outlet, and the tubing was connected to the packer. The 100 $\mathrm{mg}$ particles were suspended in $5 \mathrm{~mL}$ methanol, sonicated for $5 \mathrm{~min}$, stood calm for $20 \mathrm{~s}$, and the supernatant was decanted out to remove very fine particles. The above process was repeated three times. The sedimented particles were suspended in $1.2 \mathrm{~mL}$ methanol and fed into the reservoir of a slurry packer.

STEP 1
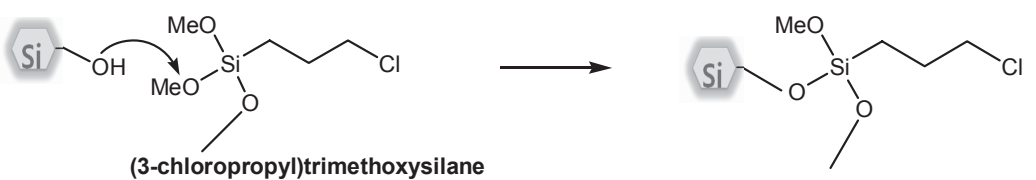

STEP 2

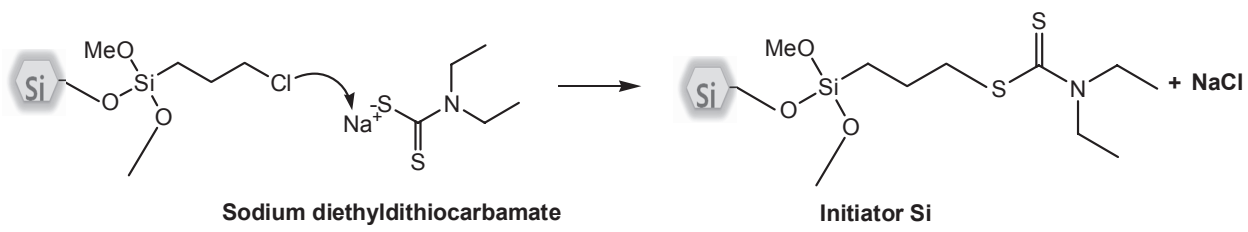

Scheme 1. The two step procedure of synthesis of the initiator attached silica monolith particles. 
The pressure of the slurry packer was instantly raised to 14,000 psi for $5 \mathrm{~min}$, adjusted to $10,000 \mathrm{psi}$ for the next $10 \mathrm{~min}$ followed by setting at $8,000 \mathrm{psi}$ for $40 \mathrm{~min}$. Then, the compressor was turned off to release the pressure on standing for a while. The column was then connected to the injector, and the 1/32 inch outlet of the column was connected to the capillary window detector by installing a graphite ferrule and a connecting capillary (50 $\mu \mathrm{m}$ I.D., $365 \mu \mathrm{m}$ O.D.). A piece of short Teflon tubing was used to connect the two capillary ends.

A test mix composed of phenol, acetophenone, 4-methyl-2nitroaniline, benzene, and toluene was prepared in acetonitrile and stored in a refrigerator at $4{ }^{\circ} \mathrm{C}$ before use. The stock sample solution was composed of $1.2 \mu \mathrm{L}$ phenol, $2 \mu \mathrm{L}$ acetophenone, $1.5 \mathrm{mg}$ 4-methyl-2-nitroaniline, $6 \mu \mathrm{L}$ benzene, and $4 \mu \mathrm{L}$ toluene in $1 \mathrm{~mL}$ solvent. The stock sample was diluted enough with the mobile phase as long as sufficient signal sensitivity was secured to avoid sample overloading. The chromatograms were obtained in 70/30 (v/v) acetonitrile/water with $0.1 \%$ TFA at a flow rate of $7 \mu \mathrm{L} / \mathrm{min}$ at $214 \mathrm{~nm}$.

\section{Results and Discussion}

Architecture of stationary phases. The microscopic view of the silica monolith particles in comparison with the SEM view is given in Figure 1. The average particle size was found $3.7 \mu \mathrm{m}$ by a Malvern (Worcestershire, UK) Mastersizer 2000 particle size analyzer. It should be noted that the monolith particles were prepared without sieving in this study. Nevertheless, some modification of the prior procedure enabled preparation of monolith particles with proper size distribution without sieving. The SEM photos of the polystyrene bound initiator silica in different view scales are compared in Figure 2, and the elemental analysis results of the initiator monolith silica, the polystyrene bound monolith silica, and the $\mathrm{C} 18$ modified monolith
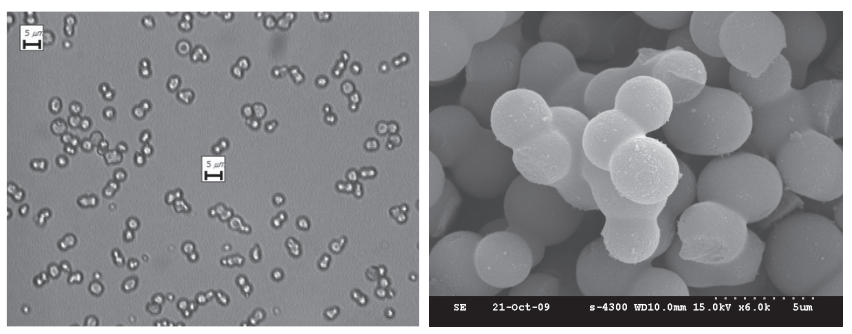

Figure 1. The images of the ground monolith silica particles taken by an optical microscopy and SEM. silica are summarized in Table 1.

In one of our previous studies ${ }^{22}$ where a more active initiator was attached to silica, the resultant stationary phase had a polystyrene layer of irregular thickness, and some lumps of aggregated polystyrene were formed probably owing to heterogeneous formation of polystyrene. Rather homogeneous polymerization was achieved with a new initiator in this study where ground monolith particles were used instead of commercial silica as shown in Figure 2. There were no large polystyrene lumps on the surface of the polystyrene bound silica. Homogeneous polymerization was also observed when the same

Table 1. Comparison of the EA results among the initiator silica monolith, the polystyrene bound initiator silica monolith, and the $\mathrm{C} 18$ bound silica monolith. ${ }^{a}$ The data for the phases obtained from the conventional silica (Lichrosorb) taken from reference 23 are given in parentheses for comparison.

\begin{tabular}{cccc}
\hline Element & $\begin{array}{c}\text { Initiator silica } \\
\text { monolith }\end{array}$ & $\begin{array}{c}\text { Polystyrene bound } \\
\text { initiator silica } \\
\text { monolith }\end{array}$ & $\begin{array}{c}\mathrm{C} 18 \text { bound silica } \\
\text { monolith }\end{array}$ \\
\hline Carbon & $3.24 \%(6.51)$ & $10.76 \%(19.96)$ & $7.12 \%(10.63)$ \\
Hydrogen & $0.75 \%(0.90)$ & $1.41 \%(1.98)$ & $1.61 \%(1.96)$ \\
\hline
\end{tabular}

${ }^{a}$ The pure weight of an element in the sample divided by the total sample weight multiplied by 100 is the \% value reported. For example, the converted pure carbon weight from the experimental result divided by the sample weight times 100 is the $\%$ carbon load.

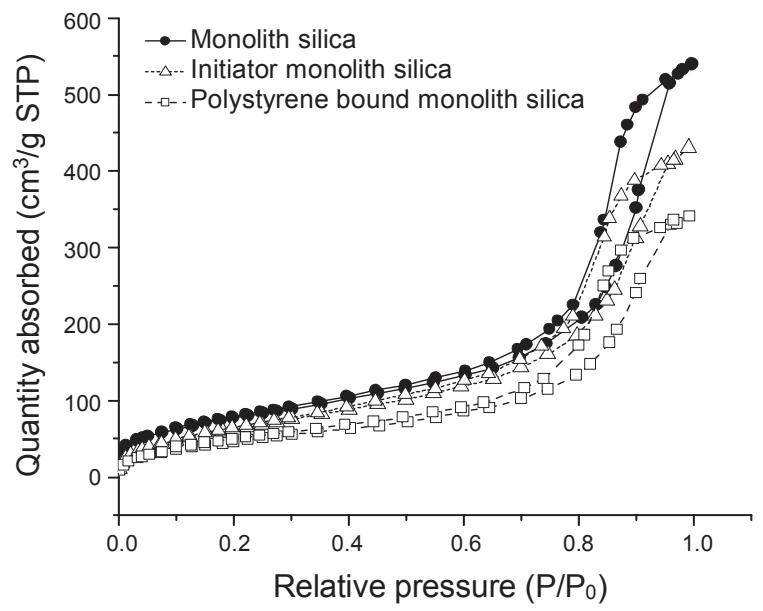

Figure 3. The $\mathrm{N}_{2}$ adsorption/desorption isotherms of the ground monolith silica, initiator attached monolith silica, and polystyrene bound monolith silica.
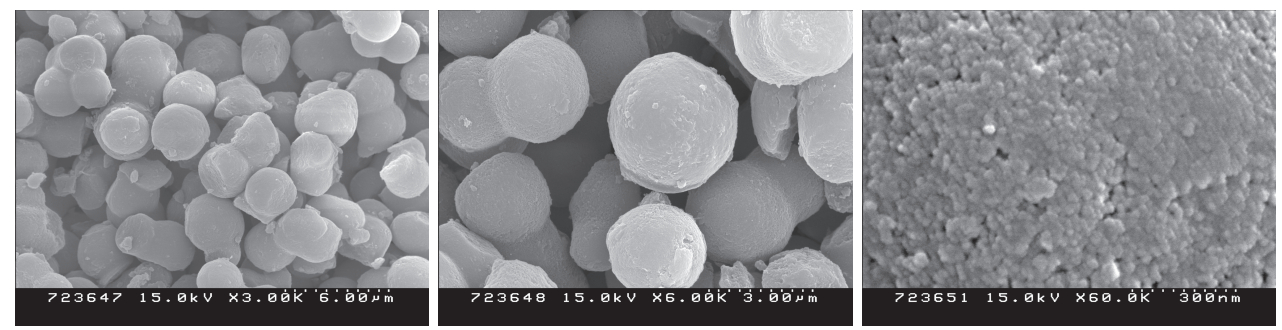

Figure 2. The SEM images of the polystyrene bound silica made from the monolith particles. From the left, the view of multiple particles, the view of single particle, and the expanded view of the surface of single particle are shown. 


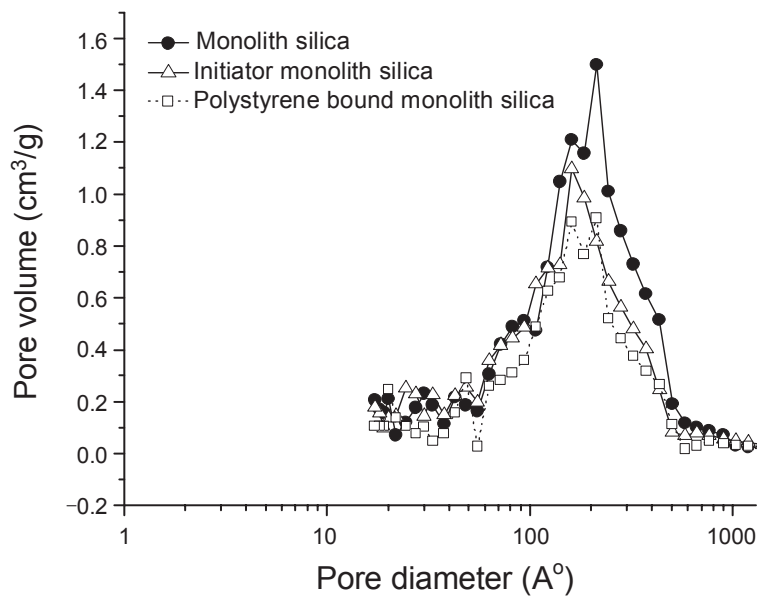

Figure 4. The BJH differential pore volume (dV/dlogD) plots against pore size for the ground monolith silica, initiator bound monolith silica, and polystyrene bound monolith silica.

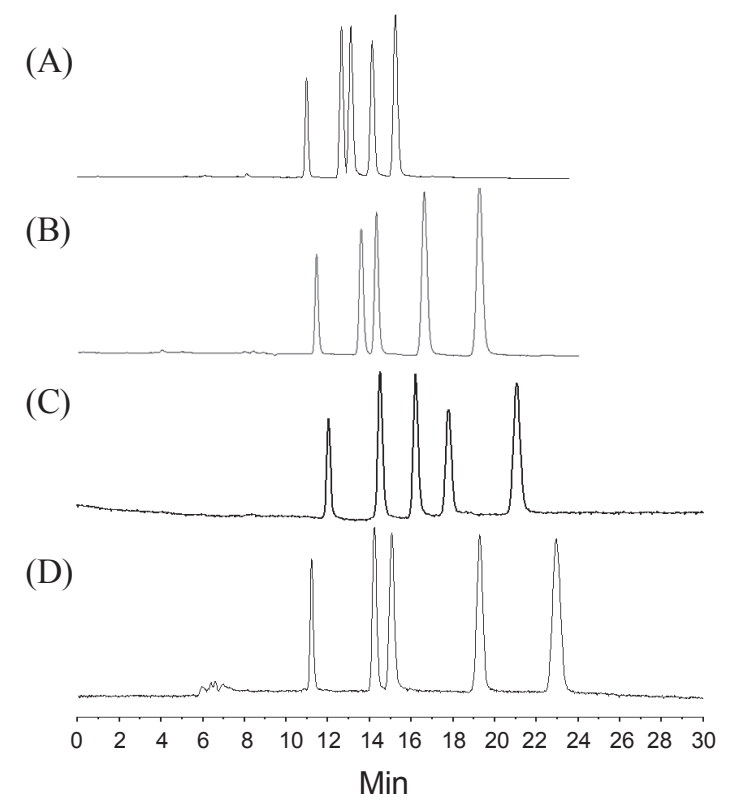

Figure 5. Comparison of chromatograms obtained by (A) polystyrene bound silica monolith particles (this study), (B) C18 bound silica monolith particles (this study), (C) C18 bound Lichrosorb particles (previous study), and (D) polystyrene bound Lichrosorb particles (previous study). The chromatograms were obtained in 70/30 (v/v) acetonitrile/water with $0.1 \%$ TFA at a flow rate of $7 \mu \mathrm{L} / \mathrm{min}$ at $214 \mathrm{~nm}$. The solutes eluted in the following order: phenol, acetophenone, 4methyl-2-nitroaniline, benzene, toluene.

initiator was attached to commercial silica. ${ }^{23-24}$

The BET/BJH $\mathrm{N}_{2}$ adsorption/desorption isotherms are shown in Figure 3, and the BJH differential pore volume $(\mathrm{dV} / \mathrm{d} \log \mathrm{D})$ distribution plots, in Figure 4, respectively, for the ground silica monolith, the initiator attached ground silica monolith, and the polystyrene attached ground silica monolith. It is clear from Figure 3 that the total pore volume was gradually decreased as the ground silica monolith was modified with the initiator and the polystyrene in sequence. Such decreasing trend of pore volume is also monitored in Figure 4. A unique observation of Figure 4 is, however, that attachment of the initiator to the gro- und silica monolith caused considerable decrease of the average pore size but additional attachment of polystyrene caused little decrease of pore size. Detailed discussion on this matter will be given in the next section.

The polystyrene attached phase of this study was stable at least for 6 months to maintain separation performance in regular mobile phase (70/30 acetonitrile/water, $0.1 \%$ TFA), but investigation in other mobile phases is required in further study.

Comparison of separation performance of the polystyrene bound silica monolith phase with otherstationary phases. Identical microcolums $(300 \times 0.5 \mathrm{~mm})$ were packed with the polystyrene combined initiator silica monolith particles and the $\mathrm{C} 18$ modified and end-capped silica monolith particles, and their chromatographic performances were examined in comparison with the prior study. The chromatographic results were compared in Figure 5. The data of number of theoretical plates $(\mathrm{N})$ were compared in Table 2.

As shown in Figure 5 and Table 2, the separation efficiency of the polystyrene bound silica monolith was found better than those of the others. The reason of superiority of a polystyrene bound phase over a $\mathrm{C} 18$ bound phase was already discussed in the previous studies ${ }^{23-24}$ and attributed to the core/shell like structure of the polystyrene bound phase. The particle of our phase is regarded to have a structure where the deep core is closed, the shallow pores are partially filled, and the outer surface is smoothly covered with bound polystyrene oligomers with relatively high molecular weights.

On the other hand, the superiority of the polystyrene bound ground silica monolith phase of this study over the polystyrene attached phase of the conventional silica should be owing to the favorable shape of the former. The silica monolith powders were composed of bent and irregular particles in addition to spherical particles, and the situation was maintained even after attachment of polystyrene layer as shown in Figure 2, thus the structure of the column packed with this phase should have some monolithic structure with flow-through channels brining about fast mass transfer kinetics and narrow bandwidths.

It should be noted that the peaks separated by the polystyrene bound silica monolith phase (Figure 5A) were more crowded than those separated by the polystyrene bound phase based on the conventional silica particles of the previous study (Figure 5D) although the separation efficiencies (numbers of theoretical plates) of the former were better than those of the latter (Table 2). It is owing to the fact that the retention time of the first eluting peak (phenol) of Figure 5A was very close to that of Figure 5D while the retention time of the last eluting peak (toluene) of Figure 5A was much earlier than that of Figure 5D. It also should be noted that the carbon loads of the phases based on the ground monolith silica of this study were considerably lower than those based on the conventional silica particles as shown in Table 1. According to Table 1, the number density of initiator molecule in the ground monolith was much smaller than that in the same amount of the conventional silica particles, which means that the number density of active silanol groups in the former should be much less than that in the latter.

Some particular features of Table 1 should be noted. First of all, the carbon load (and hydrogen content, too) of any phase of this study was less than that of the previous study. It implies that 
Table 2. Comparison of the values of number of theoretical plates (averages and standard deviations) based on 3 batches of micro columns $(0.5 \times$ $300 \mathrm{~mm}$ ) among various phases. The chromatograms were obtained in $70 / 30(\mathrm{v} / \mathrm{v})$ acetonitrile/water with $0.1 \% \mathrm{TFA}$ at a flow rate of $7 \mu \mathrm{L} / \mathrm{min}$ at $214 \mathrm{~nm}$.

\begin{tabular}{ccccc}
\hline Solute & $\begin{array}{c}\text { Polystyrene bound } \\
\text { monolith silica }\end{array}$ & $\begin{array}{c}\text { C18 bound } \\
\text { monolith silica }\end{array}$ & $\begin{array}{c}\text { Polystyrene bound } \\
\text { conventional silica }^{a}\end{array}$ & $\begin{array}{c}\text { C18 bound } \\
\text { conventional silica }^{a}\end{array}$ \\
\hline phenol & $29800 \pm 300$ & $27000 \pm 500$ & $26000 \pm 500$ & $21200 \pm 600$ \\
acetophenone & $28100 \pm 400$ & $26900 \pm 600$ & $24100 \pm 400$ & $20300 \pm 400$ \\
4-methyl-2-nitroaniline & $26400 \pm 800$ & $29700 \pm 900$ & $22000 \pm 900$ & $22100 \pm 800$ \\
benzene & $31900 \pm 400$ & $23800 \pm 500$ & $21800 \pm 400$ & $20800 \pm 500$ \\
toluene & $31100 \pm 500$ & $24600 \pm 600$ & $19500 \pm 800$ & $21100 \pm 600$ \\
Average & $29500 \pm 2200$ & $26400 \pm 2300$ & $22700 \pm 2500$ & $21100 \pm 700$ \\
\hline
\end{tabular}

${ }^{a}$ Data taken from reference 23 .

the surface silanol group density of the silica monolith particles of this study is lower than that of the conventional spherical silica particles.

Next, the carbon content increase for the polystyrene attachment was greater than that for the $\mathrm{C} 18$ attachment while the hydrogen content increase for the $\mathrm{C} 18$ attachment was greater than that for the polystyrene attachment. It is owing to the fact that the relative hydrogen content of polystyrene is smaller than that of $\mathrm{C} 18$ ligand since $\mathrm{H}: \mathrm{C}$ mol ratio is close to 1 in polystyrene while it is close to 2 in $\mathrm{C} 18$.

It is suspected that the hydrogen content of initiator attached silica (both monolith and conventional) was quite overestimated since it was air-dried at room temperature and subject to humidity absorption. Air-drying was carried out in order to prevent thermal decomposition since polystyrene would not be attached after initiator decomposition. On the other hand, the ligand attached phases were dried at $60{ }^{\circ} \mathrm{C}$ for at least $10 \mathrm{~h}$.

The above factors also seem to be the reason for the observation that the difference in carbon content between the monolith silica based phases of this study and the phases based on the conventional silica was rather high while the difference in hydrogen content was low.

The peak pore size in the differential pore volume $(\mathrm{dV} / \mathrm{d} \operatorname{logD})$ distribution plot $\left(212 \mathrm{~A}^{\circ}\right.$, Figure 4$)$ of the ground monolith was larger than that $\left(146 \mathrm{~A}^{\circ}\right)$ of the conventional silica partilcles. ${ }^{23}$ However, the total pore volume and the BET specific surface area of the ground monolith were found $0.83 \mathrm{~cm}^{3} / \mathrm{g}$ and $283 \mathrm{~m}^{2} / \mathrm{g}$, and those of the conventional silica, $0.64 \mathrm{~cm}^{3} / \mathrm{g}$ and $161 \mathrm{~m}^{2} / \mathrm{g}$, respectively. Thus, the properties of the two substrates are totally different in such way that the ground monolith silica has a wider surface area but a much lower density of surface silanol groups than the conventional silica. As shown in Figure 4, the pore size was considerably decreased by attachment of the initiator to the ground silica, but little change was observed by further attachment of polystyrene. The total pore volume of the ground silica monolith, initiator attached silica monolith, and polystyrene bound silica monolith were found $0.83,0.66$, and $0.53 \mathrm{~cm}^{3} / \mathrm{g}$, while their carbon load values were $0,3.24$, and $10.76 \%$, respectively (Table 1 ). In other words, the pore volume was decreased by $0.17 \mathrm{~cm}^{3} / \mathrm{g}$ and the carbon load was increased from 0 to $3.24 \%$ for the initiator attachment, while the pore volume was decreased by only $0.13 \mathrm{~cm}^{3} / \mathrm{g}$ and the carbon load was increased from 3.24 to $10.76 \%$ for the next polystyrene attachment. Two reasons may be responsible for this. First, the inner pores were clogged by polymeriazation. ${ }^{23-24}$ Second, majority of polymerization was taken place on the outer surface of each particle and inner surface polymerization was minimized for poor spatial accessibility and low density of surface attached initiator molecules.

The unique characteristics of the new silica monolith powders (high total pore volume and low silanol density) have their own advantages and disadvantages. The main disadvantage is, of course, the low carbon load of the final modified phase. However, the low surface silanol group density is expected to result in few residual silanol groups in the final phase minimizing adsorption band broadening as demonstrated by the high separation efficiency of this study. Furthermore, the silica monolith powders of this study have relatively large pores to suggest a prospective improvement where the carbon load of the polystyrene bound phase may be increased with a proper choice of initiator to result in real core-shell type phases without an adsorption problem (complete coverage of residual silanol groups) appropriate for separation of proteomic samples. Such study is under way.

\section{Conclusion}

A new stationary phase has been prepared by attaching an initiator to ground silica monolith particles and forming polystyrene on the silica surface by reversible addition-fragmentation chain transfer polymerization. A modified preparation method of ground silica monolith particles was developed to yield the monolith particles with proper size distribution without sieving. The separation efficiency of the new stationary phase was better than that of the polystyrene attached phase on the conventional silica particles.

Acknowledgments. This work was supported by the Inha University Fund.

\section{References}

1. Rahman, M. M.; Czaun, M.; Takafuji, M.; Ihara, H. Chem. Eur. J. 2008, 14, 1312-1321.

2. Nagase, K.; Kobayashi, J.; Kikuchi, A.; Akiyama, Y.; Annaka, M.; Kanazawa, H.; Okano, T. Langmuir 2008, 24, 10981-10987.

3. Idota, N.; Kikuchi, A.; Kobayashi, J.; Akiyama, Y.; Sakai, K.; 
Okano, T. Langmuir 2006, 22, 425-430.

4. Coad, B. R.; Steels, B. M.; Kizhakkedathu, J. N.; Brooks, D. E.; Haynes, C. A. Biotechnol. Bioengineer. 2007, 97, 574-587.

5. Nagase, K.; Kobayashi, J.; Kikuchi, A.; Akiyama, Y.; Kanazawa, H.; Okano, T. Langmuir 2008, 24, 511-517.

6. Mallik, A. K.; Rahman, M. M.; Czaun, M.; Takafuji, M.; Ihara, H. J. Chromatogr. A 2008, 1187, 119-127.

7. Hemstrom, P.; Szumski, M.; Irgum, K. Anal. Chem. 2006, 78, 70987103.

8. Miller, M. D.; Baker, G. L.; Bruening, M. L. J. Chromatogr. A 2004, 1044, 323-330.

9. Yoshikawa, C.; Goto, A.; Tsujii, Y.; Ishizuka, N.; Nakanishi, K.; Fukuda, T. J. Polym. Sci. Part A: Polym. Chem. 2007, 45, 47954803.

10. Nagase, K.; Kobayashi, J.; Kikuchi, A.; Akiyama, Y.; Kanazawa, H.; Okano, T. Biomacromolecules 2008, 9, 1340-1347.

11. Derouet, D.; Thuc, C. N. H. J. Appl. Polym. Sci. 2008, 109, $2113-$ 2127.

12. Fairhurst, R. E.; Chassaing, C.; Venn, R. F.; Mayes, A. G. Biosensors and Bioelectronics 2004, 20, 1098-1105.

13. Sulitzky, C.; Ruckert, B.; Hall, A. J.; Lanza, F.; Unger, K.; Sellergren, B. Macromolecules 2002, 35, 79-91.

14. Roohi, F.; Titirici, M. M. New J. Chem. 2008, 32, 1409-1414.
15. Su, S.; Zhang, M.; Li, B.; Zhang, H.; Dong, X. Talanta 2008, 76, 1141-1146.

16. Czaun, M.; Rahman, M. M.; Takafuji, M.; Ihara, H. Polymer 2008, 49, 5410-5416.

17. Unsal, E.; Elmas, B.; Caglayan, B.; Tuncel, M.; Patir, S.; Tuncel, A. Anal. Chem. 2006, 78, 5868-5875.

18. Coessens, V.; Pintauer, T.; Matyjaszewski, K. Prog. Polym. Sci. 2001, 26, 337-377.

19. Qiu, K.; Li, P. Chinese J. Polym. Sci. 2004, 22, 99-110.

20. Favier, A.; Charreyre, M. Macromol. Rapid Comm. 2006, 27, 653692.

21. Perrier, S.; Takolpuckdee, P. J. Polym. Sci. Pt. A: Polym. Chem. 2005, 43, 5347-5393.

22. Kim, S. S.; Cheong, W. J. Bull. Korean Chem. Soc. 2009, 30, $722-$ 725 .

23. Hwang, D. G.; Zaidi, S. A.; Cheong, W. J. Bull. Korean Chem. Soc. 2009, 30, 3127-3130.

24. Hwang, D. G.; Zaidi, S. A.; Cheong, W. J. J. Sep. Sci. 2010, 33, $587-593$.

25. Ko, J. H.; Baik, Y. S.; Park, S. T.; Cheong, W. J. J. Chromatogr. A 2007, 1144, 269-274.

26. Han, K. M.; Cheong, W. J. Bull. Korean Chem. Soc. 2008, 29, 2281-2283. 